\title{
Neuroscientists claim growing pains
}

\section{Manufacturer denies that common lab feed can cause some neuron cultures to fail.}

Leading neuroscientists are warning that difficulties with a staple laboratory product may be costing time and money.

The scientists say that variation between batches of a growth medium designed to sustain neurons in culture can, in their experience, cause experiments to fail or give low-quality results because of the poor survival and maturation of cells.

The growth medium in question is a particular formulation of B27, a mixture of proteins, hormones and vitamins, produced by laboratory supplies company Invitrogen of Carlsbad, California, now a division of Life Technologies. In 2004, a group of neuroscientists including
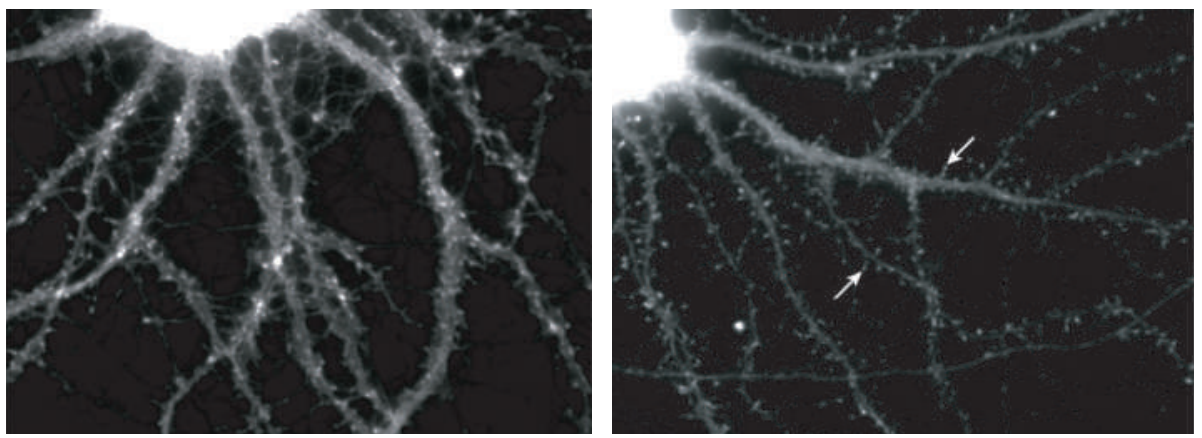

Culture differences: dendritic spines (arrowed) are signs of development that were visible after neurons were cultured for 18 days in NS21 (right), but not in B27 (left), in Johannes Hell's laboratory. Beth Stevens, now at Harvard Medical School, in Boston, Massachusetts, and Johannes Hell of the University of Iowa, Iowa City, told Invitrogen that they thought the $\mathrm{B} 27$ medium was producing poor results following a change in the product's ingredients. The company says that it remedied the problem, and that the number of complaints it received from scientists fell to "negligible levels".

"Things were improved," says Stevens, "but over the next few years it became clear there is still a lot of variation in quality control." Hell estimates that his lab spends about US $\$ 50,000$ per year on culturing neurons. "If you get mediocre cultures, that money is wasted," he says.

So Hell and other colleagues developed an alternative growth medium called Neuronal Sup-

\section{"If you get mediocre cultures, money is wasted."}

NS21, particularly at lower cell densities. "That was a clear effect for me," says Harata. "I've now completely switched to using NS21."

Hell says that in his capacity as a reviewer he has seen academic papers in which he suspects poor-quality cultures may be due to problems with B27. Oliver Schlüter, of the European Neuroscience Institute in Göttingen, Germany, agrees that there is "batch-to-batch variation" in B27. In his own lab, "we test the batches and compare them with what we had previously", Schlüter says. "If it behaves similarly we buy as much as we can."

Joydeep Goswami, vicepresident of primary and stem-cell systems at Life Technologies, says that batches of B27 can vary because the medium includes ingredients plement 21 (NS21), which they unveiled last year (Y. Chen et al. J. Neurosci. Meth. 171, 239$247 ; 2008)$. Hell points out that NS21 is not a commercial product; the researchers who use it prepare it in their own laboratories.

Charles Harata, a colleague of Hell at the University of Iowa who was not involved in the development of NS21, used B27 while working as a postdoc for Richard Tsien at Stanford University, California. Harata felt that the quality of his hippocampal-neuron cultures declined markedly in the years before he left Tsien's lab in 2007.

That summer he took up a full-time post at Iowa, initially using B27. About six months ago, however, he decided to try his new colleague Hell's NS21 formulation.

Harata conducted a blind comparison of B27 and NS21 to grow two neuron cultures in otherwise identical conditions. He found that B27 did not support the neurons as well as from biological sources, such as bovine serum albumin, that can be difficult to standardize. $\mathrm{He}$ adds that each batch goes through a rigorous quality-control process, assessed periodically by the US Food and Drug Administration, and "the variability should not affect customers that are following standard protocols".

Goswami asserts forcefully that B27 is not wasting researchers' time and money, adding, "We stand behind our products."

He suggests that variability in results may arise not from B27 itself but from the different types of cell grown in the medium, or the different procedures used by scientists running the experiments.

The neuroscientist who originally developed B27, Gregory Brewer, works at Southern Illinois University, Springfield, and is a consultant for Invitrogen. He points out that his original studies tested B27 on its ability to sustain neurons for four days. Now, he says, neuroscientists often expect the medium to keep neurons in shape for weeks, the time period after which features such as synapses and dendritic spines appear (see pictures of hippocampal neurons from rat embryos, above).

Brewer still uses B27 himself, and intends to discuss with Invitrogen an assessment of whether the medium can support extended growth times.

Richard Haigh, director of product development for Invitrogen, agrees that B27 "is now used in a broad range of applications beyond its original purpose" and says that the company is looking at products that might better support researchers' specific cell types.

\section{Replication challenge}

The precise makeup of Invitrogen's B27 is commercially sensitive, although the ingredients of the original B27 formulation are listed in the research paper that first defined the medium (G. Brewer et al. J. Neurosci. Res. 35, 567-576; 1993). "My biggest concern is that we're dealing with a black-box chemical," says Schlüter. "If you want to replicate other people's experiments, you need to know what's in there and what variation could happen."

Goswami points out that customers can get technical help from Invitrogen and can request data sheets for each batch.

Yet Stevens believes that scientists having problems with their neuron cultures may not even question if their growth medium could be a factor. "I'm quite sure people are banging their heads against a wall. Trying to troubleshoot this can waste months," she says.

"Frankly we don't hear the same thing," counters Goswami. "More than 1,000 labs are using B27 successfully."

Daniel Cressey 